\section{|||||||||||||||||||||||||||||||||||||||||||||||||||||||||||||||||||||||.}

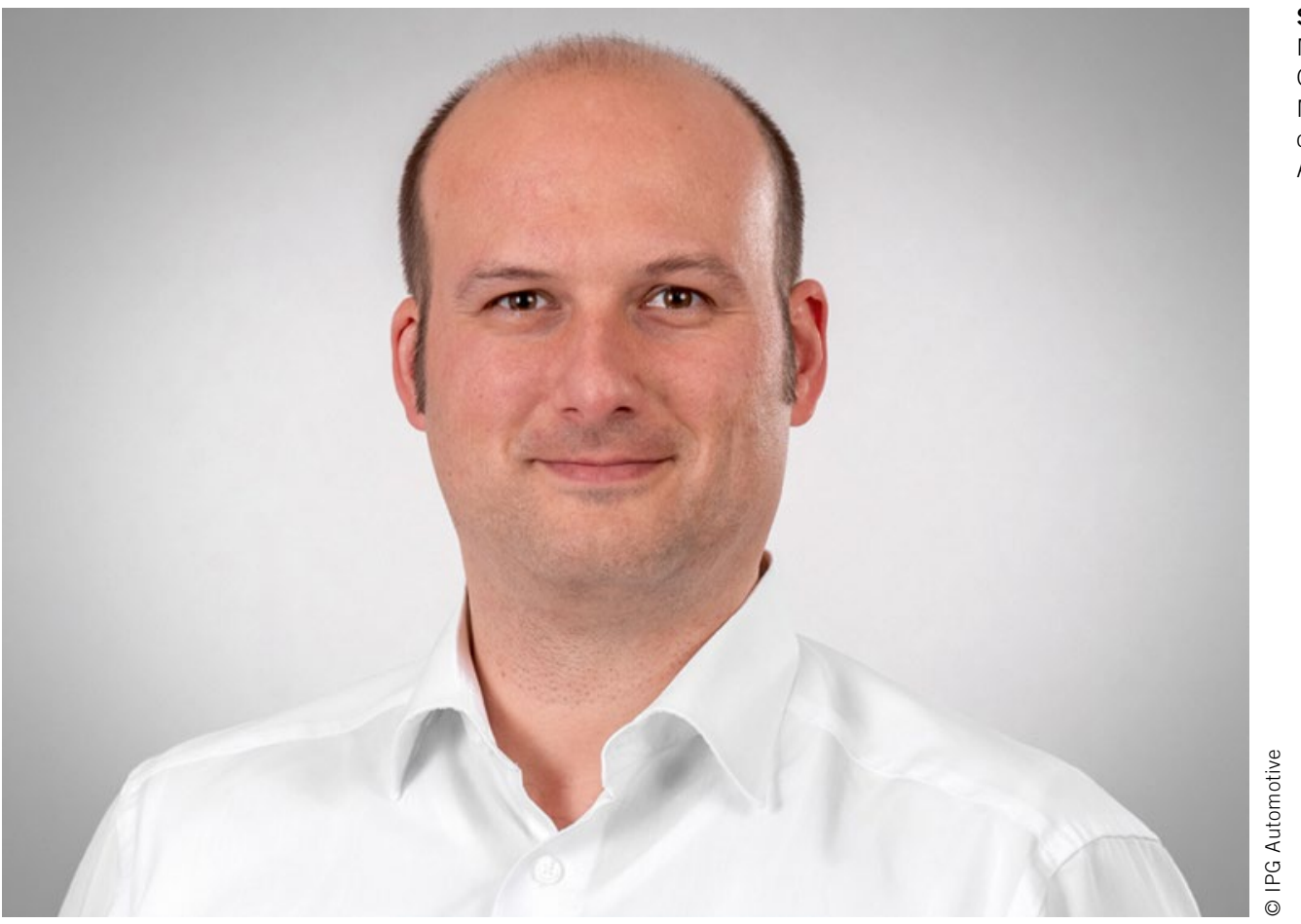

Steffen Schmidt

Managing Director IPG Automotive $\mathrm{GmbH}$ in Karlsruhe (Germany), Member of the Advisory Board of the ATZlive Conference Automated Driving

\title{
How CAE Can Learn from CAD
}

Product lifecycle management and CAD systems provide an important infrastructure for automotive development. They enable a centrally maintained data management that is standardized throughout the entire development process. From the first design sketches to production, all partners of a development project work on a shared database. This infrastructure is essential to manage the complexity of current vehicles.

Due to the increasing complexity of mechatronic systems and the rapid increase in software development efforts, simulation plays a crucial role in development processes. Simulation today is a driver for innovation in vehicle development. It allows for high quality virtual prototypes that can be used across multiple domains to develop, calibrate, test and release individual mechatronic systems or system networks in the full vehicle at every development stage along the V-model. The high accuracy in tests leads to quality improvement, and the early optimization of components and systems with virtual tests shortens development times. In addition, front-loading and the reduced need for physical prototypes lower costs.

Nevertheless, the use of simulation is not widely common: The deployed tools also need functional data for parameterization. Today, these data are often not filed and maintained centrally. Accordingly, simulation is generally used as an isolated solution. Cross-domain systems engineering can benefit tremendously from integrating simulation in the entire develop- ment process. A necessary requirement is to establish an infrastructure that adopts the characteristics of the tools and workflows of the CAD world and adapts to the special requirements of simulation at the same time.

Core elements are a structured management of the diverse data and models necessary for simulation, paired with a verifiable quality assurance. Considering that and the provision of simulation models and test scenarios, dedicated organizational units need to be established and developed. They are responsible for the tool chain, coordinate $\mathrm{CAE}$ activities in the entire company and ensure the exchange of models with suppliers and the integration of software in the versioned simulation models.

To establish valid decisions based on simulation results and gain advantages in quality, time and cost efficiency in the entire company, adapting the development processes is necessary. I am confident that the organizations, processes and tool chains adjusted to the complexity of automotive software will gradually find their way into automotive development, and strengthen the collaboration between the different domains. An infrastructure is thereby established that enables systems engineering as well as an evaluation of development steps long before physical prototypes are available. This development does not halt at company boundaries, it will redefine collaborations between OEMs and Tier-1 suppliers. 